\title{
EVALUATING THE RISK OF ADVERSE EVENTS IN HOSPITAL SECTOR THROUGH HYBRID MODEL AHP-DEMATEL-VIKOR METHODS
}

\begin{abstract}
The aim of the present paper is to propose a hybrid model with AHP, DEMATEL and VIKOR methods to assess the risk of adverse events in a hospital. AHP has been used to determine the criteria and sub-criteria weights, and then DEMATEL (Decision Making Trial and Evaluation Laboratory) has been employed to calculate interrelations between criteria and finally, VIKOR method ranks the 3 hospitals from Healthcare Sector according to the risk of adverse events.
\end{abstract}

Keywords: Healthcare, VIKOR, DEMATEL, AHP, adverse events

\section{Introduction}

Patient safety is considered as a fundamental critical to satisfaction in healthcare (Martins et al., 2011). Nevertheless, there could have errors that can cause injury or death. These errors can be detected before occurring in healthcare services; but some of them are not detected and might cause damage in patient's health. If this error brings about damage, is called adverse event. Unfortunately, in Colombia, adverse events are frequent and cause death in some cases.

\section{Literature Review}

Many articles have been written about applying decision support systems to medical and health care decision making but little has been published about the complex problem of patient safety (Liberatore and Nydick, 2008; De Felice and Petrillo, 2015). This problem has become the domain of medical assessment. To help people make better decisions in these circumstances, a number of multi-criteria decision-making methods have been developed (Dolan, 2008).

\section{Hypotheses/Objectives}

As stated by Martins et al. (2011), the occurrence of adverse events is considered a serious problem worldwide regarding the performance of health services. Considering the relevance of this theme and the lack of studies, this research aims to evaluate the risk of adverse events in hospitalized patients in Colombia. 
ISAHP Article: A Style Guide for Paper Proposals To Be Submitted to the International Symposium on the Analytic Hierarchy Process 2016, London, U.K.

\section{Research Design/Methodology}

The novelty of the present study is based on the integration of the AHP, perhaps the most well-known and widely used multi-criteria method with Decision-Making Trial and Evaluation Laboratory (DEMATEL) and VIKOR method to identify key success factors of hospital service quality in order to avoid adverse events for patients.

\section{Data/Model Analysis}

The aim of this study is to assess the risk of adverse events in a hospital. A decision making model with 7 factors, 30 sub-factors and 3 hospitals, has been designed with the support of experts from healthcare sector and the guidelines issued by London Protocol and studies developed by Ministry of Health and Social Protection (Figure 1). 


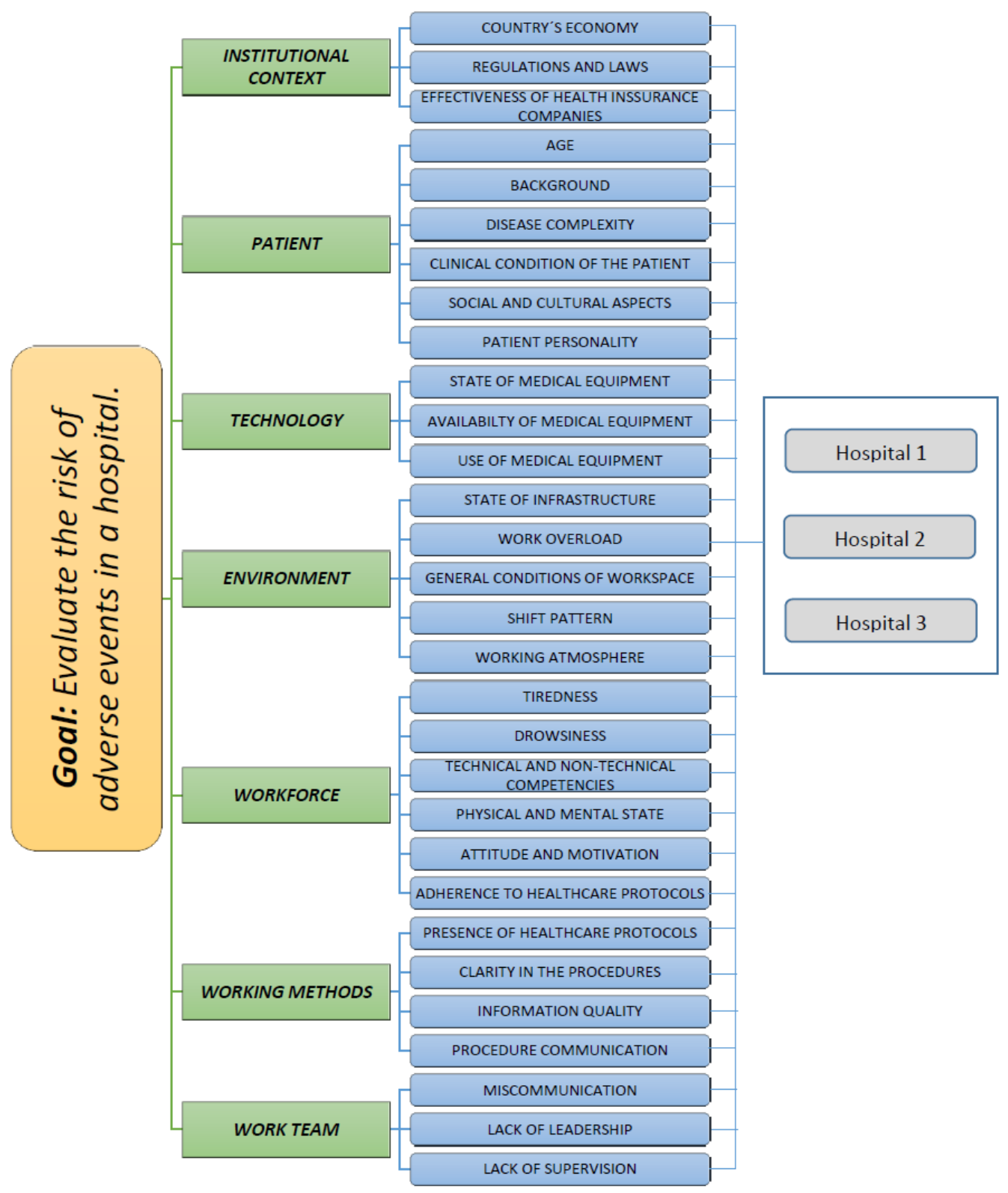

Figure 1: The proposed AHP model

The results show that the most representative factors upon evaluating the risk of adverse events in hospital sector are: PATIENT ( $\mathrm{GW}=24.8 \%)$, ENVIRONMENT $(\mathrm{GW}=$ 18.2\%), INSTITUTIONAL CONTEXT $(\mathrm{GW}=15.7 \%)$ and WORKFORCE $(\mathrm{GW}=$ $12.9 \%)$. There is not a leading factor. The contributions between factors are similar with a maximum difference of $16.7 \%$. This implies creating multi-criteria strategies to reduce the risk of adverse events in hospital. The top-ten of the representative sub-factors upon 
evaluating the risk of adverse events in hospital sector are: AGE OF PATIENT (GW = 7.2\%), COUNTRY'S ECONOMY (GW $=6.3 \%)$, GENERAL CONDITIONS OF WORKSPACE $(\mathrm{GW}=5.7 \%)$.

\section{Limitations}

It is important to acknowledge that the findings may be related to the characteristics of the study design. Importantly, the study was limited to 3 hospitals in Colombia, which could partially explain the results. Future research will be take into account two new aspect: 1) a greater number of hospitals and 2) different countries.

\section{Conclusions}

In the context of health care, the evaluation of any outcome measure involves several parameters. Thus, it is necessary to take into account the relationships between them. At this aim concur the multi criteria decision methods. The promising results obtained in applying the AHP-DEMATEL-VIKOR method suggest that the AHP and similar methods can be used to create decision aids that will simplify and expedite the shared decisionmaking process.

\section{Key References}

De Felice, F., Petrillo A., (2015). Improving Italian healthcare service quality using analytic hierarchy process methodology. $6^{\text {th }}$ European Conference of the International Federation for Medical and Biological Engineering, MBEC 2014; Dubrovnik; Croatia; 7 September 2014 through 11 September 2014, Volume 45, Pages 981-984..

Dolan, J.G., (2008). Shared decision-making - transferring research into practice: The Analytic Hierarchy Process (AHP). Patient Education and Counseling, 73, 418-425.

Liberatore, M.J., Nydick, R.L., (2008). The analytic hierarchy process in medical and health care decision making: A literature review. European Journal of Operational Research, 189, 194-207

Martins, M., Travassos, C., Mendes, W., Pavão, A,L.B., (2011). Hospital deaths and adverse events in Brazil. Health Services Research, 11,223. 